\title{
Strain differences in the mating behavior of Sprague-Dawley, Long-Evans, and Wistar male rats
}

\author{
JACK H. McLEAN, WALTER A. DUPEIRE III, and S. THOMAS ELDER \\ Louisiana State University, New Orleans, La. 70122
}

Mating tests were run to compare the level of copulatory behavior in three strains of male rats: Sprague-Dawley (SD), Long-Evans (LE), and Wistar (W). Twenty-four males served in each of the above strains and were randomly assigned to one of three groups, one that was tested with SD lures, one with LE lures, and one with $W$ lures. The $W$ males exhibited significantly more copulatory responses than the SD or LE males, while the latter two strains did not differ from each other. The $W$ females, however, were the least effective lures, eliciting significantly fewer responses than the SD or LE females.

Although the rat is commonly used as an experimental $S$ in psychology, relatively few behavioral data have been reported on differences between strains. Apparently, it is assumed implicitly that a given experimental treatment will produce a given effect regardless of the strain of rat. There are, however, a few studies which indicate behavioral strain differences. For example, strain differences have been reported for such dependent variables as hoarding (Stamm, 1954), aggression (Stamm, 1955), activity and exploration (Carr \& Williams, 1957), avoidance learning (Schaefer, 1959), emotionality (Wilcock \& Broadhurst, 1967), and instrumental behavior (Pieper, Garwood, Lewis, \& Marx, 1962).

One research area where the effects of strain are particularly lacking is sexual behavior. Intuitively, it would seem that sexual behavior in the rat would be particularly sensitive to genetic influences, since the mating pattern is so highly stereotyped. One report, Whalen (1961), did deal with the effects of strain on mating behavior and found strain to be a significant variable. The strains investigated by Whalen, however, are unfortunately not commonly used in psychological laboratories. In fact, they are quite uncommon, i.e., Tyron Maze Bright, Tyron Maze Dull, Roderick-Demster Cholinesterase $\mathrm{High}$, and Roderick-Demster Cholinesterase Low. Specifically, the major finding was that the Tyron Maze Bright Ss were able to achieve complete intromissions on a higher percentage of attempts than did the Tyron Maze Dull Ss, demonstrating a greater "copulatory efficiency."

There were two major purposes of the present research: (1) to compare the vigor of male mating behavior in three strains of rats commonly used in ps y chological research: Sprague-Dawley (SD), Long-Evans hooded (LE), and Wistar (W); and
(2) to determine the effect of strain of female lure upon male responsiveness. SUBJECTS

Twenty-four SD, $24 \mathrm{LE}$, and $24 \mathrm{~W}$ male rats, 110 days old at the onset of testing, served as Ss. Eight females from each of the above strains served as lures. All Ss were obtained from Research Animals Inc., Braddock, Pa. The Ss were housed individually and were maintained ad lib on standard Lab Chow and water.

\section{APPARATUS}

The sex tests were run in three Plexiglas-fronted semicircular arenas, $56 \mathrm{~cm}$ across the front, $38 \mathrm{~cm}$ from front to back, and $33 \mathrm{~cm}$ high. The floors of the test chambers were covered in wood shavings. The test chambers were located in a dimly illuminated sound-attenuated experimental room.

\section{PROCEDURE}

The female lures, previously ovariectomized, were brought into estrus by administering a 6.6 microgram intramuscular injection of estradiol benzoate in $0.05 \mathrm{cc}$ of sesame seed oil $54 \mathrm{~h}$ prior to a test session. A primer injection of $0.5 \mathrm{mg}$ of progesterone in $0.05 \mathrm{cc}$ of sesame seed oil was given $6 \mathrm{~h}$ prior to testing.

The 24 males in each strain were assigned randomly to one of three groups, one that was tested with SD lures, one that was tested with LE lures, and one that was tested with W lures. On each test day, nine males, three of each strain, were paired with nine lures, three of each strain, in a factorial fashion, so that each strain of male was tested with each strain of female. On any given day, no lure was placed in contact with more than one male.

Males were given $5 \mathrm{~min}$ of adpatation in the testing chamber before introducing the female. The testing session began upon introduction of the female and lasted $30 \mathrm{~min}$

The number of mounts, intromissions, and ejaculations was recorded for each $S$ during two sessions, separated by 5 days. Separate analyses were performed on each of the three dependent measures as well as on a composite sex score obtained by adding the frequencies of mounts, intromissions, and ejaculations. The analysis of variance design consisted of three factors with repeated measures on one factor. Duncan's new multiple range test and $F$ tests for simple effects were used subsequent to statistically significant $F$ values.

\section{RESULTS AND DISCUSSION}

The three measures of sexual activity (number of mounts, number of intromissions, and number of ejaculations) yielded the same $F$ values statistically significant in analyses of variance. In view of the fact that one would reach the same conclusions regardless of which dependent variable is considered, the analyses reported here are based on the composite sex score.

The main effect due to strain of male was statistically significant beyond the .01 level $(\mathrm{F}=12.5, \mathrm{df}=$ 2/63). The $W$ males exhibited significantly $(\mathrm{p}<.01)$ more copulatory responses than either the SD or LE Ss (means were 23.16, 12.23 , and 10.06, respectively). As one may see in Fig. 1 , the W Ss were the most vigorous males for all three strains of lure. The SD and LE males did not differ from each other.

The main effect due to strain of female was also statistically significant beyond the $1 \%$ level $(\mathrm{F}=11.75, \mathrm{df}=$ $2 / 63)$. Whereas the $W$ males were the most vigorous of the male strains, the $\mathrm{W}$ females were the least effective lures, eliciting significantly $(p<.01)$ fewer copulatory responses than SD or LE females (means were $7.52,17.33$, and 20.60 , respectively).

The final statistic of interest is the Strain of Male by Strain of Female interaction. This source of variance was significant beyond the .01 level ( $F$ $=4.23$, $\mathrm{df}=4 / 63$ ). This interaction may be interpreted to mean that the effect of strain of lure was different for different strains of males. That is, the effect of female strain depended upon which strain of male was being considered. In order to examine this interaction more closely, $F$ tests for simple effects were run, holding male strain constant at one level (one strain) and testing for differences across the three female strains. These tests showed that there were statistically significant differences between the number of responses elicited by the female strains for both the SD and W males $\left(\mathrm{Fs}_{\mathrm{s}}=7.52\right.$ and 9.81 , respectively, $\mathrm{df}=2 / 63$ ). There were no differences, however, between the three female strains when considering the LE males $(F=0.24, \mathrm{df}=2 / 63)$. 


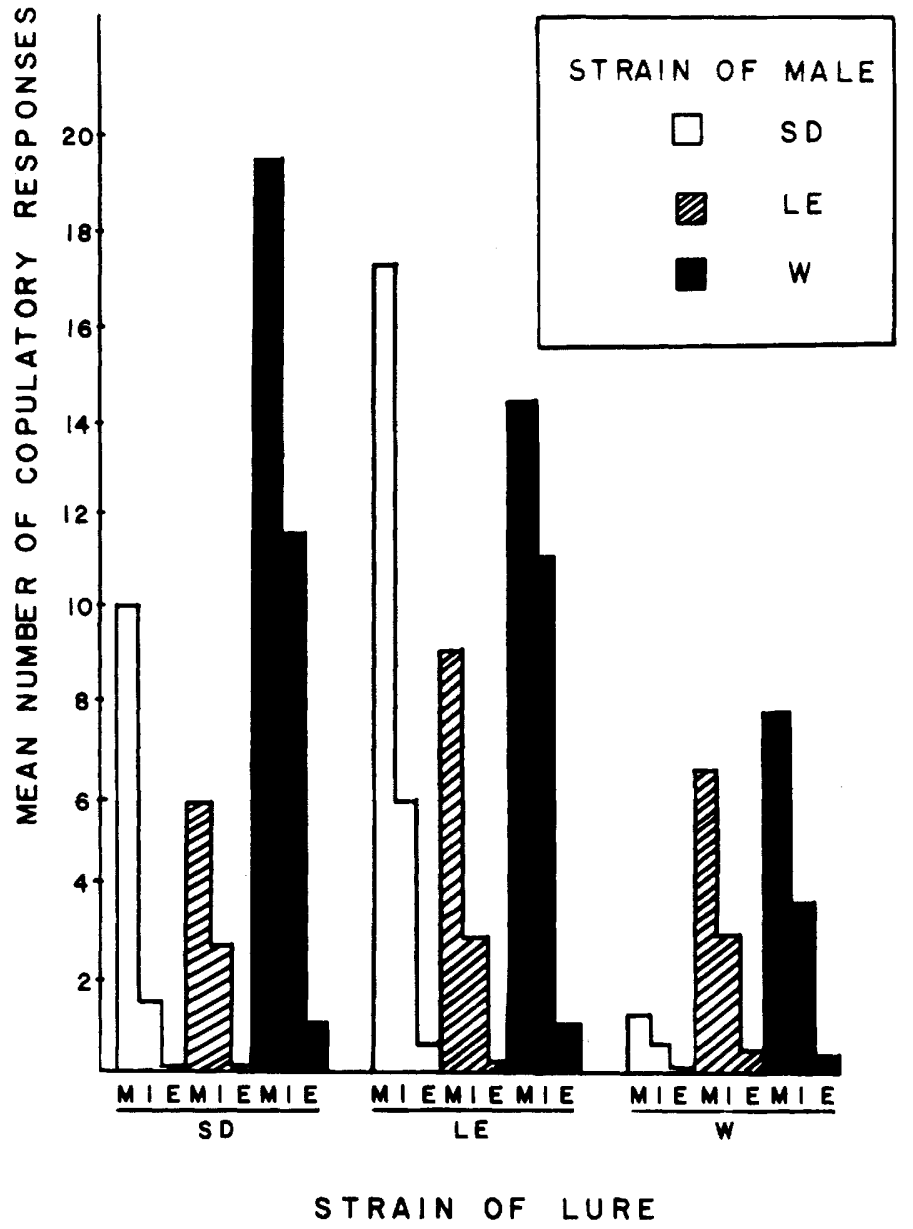

Fig. 1. Mean number of mounts (M), intromissions (I), and ejaculations (E), for all three strains of male tested with each strain of female. Each bar represents the mean of eight Ss over two test sessions.

The results of these simple effects are reflected in Fig. 1. Note that the number of copulatory responses varies considerably from one strain of female to another for the SD males, with the LE lures being the most preferred and the $\mathrm{W}$ lures the least. For the $\mathrm{W}$ males, also, strain of female was important in determining number of responses were approximately equally preferred, with the $W$ females a substantial emitted. Here, the SD and LE females distance behind. For the LE males, however, the strain of lure was unimportant in determining the number of copulatory responses. These Ss mated at about the same level regardless of the strain of female.

None of the within-S sources of variance was significant.

It should be emphasized that the statistically significant differences reported here are on an organismic variable, and these strains may differ from one another in several respects other than copulatory behavior. For example, one might investigate strain differences on such dependent measures as activity, social dominance, aggression, hormone levels, sex pheromones, etc.

\section{REFERENCES}

CARR R M \& WILLIAMS, C D. Exploratory behavior of three strains of rats. Journal of Comparative \& Physiological Psychology, 1957, 50, 621-623.

PIEPER, W. A., GARWOOD, C., LEWIS, D., \& MARX, M. H. Differential acquisition of an instrumental response in albino and hooded rats. Psychological Reports, $1962,11,425-426$.

SCHAEFER, V. H. Differences between strains of rats in avoidance conditioning without an explicit warning stimulus. Journal of Comparative \& Physiological Psychology, 1959, 52, 120-122.

STAMM, J. S. Genetics of hoarding: I. Hoarding differences between homozygous strains of rats. Journal of Comparative \& Physiological Psychology, $1954,47,157-161$.

STAMM, J. S. Hoarding and aggressive behavior in rats. Journal of Comparative \& Physiological Psychology, 1955, 48, 324-326.

WHALEN, R. E. Strain differences in sexual behavior of the male rat. Behavior,1961, 18, 199-204.

WILCOCK, J., \& BROADHURST, P. L. Strain differences in emotionality: Open-field and conditioned avoidance behavior in the rat. Journal of Comparative \& Physiological Psychology, $1967,63,335-338$. 